\title{
Wavelet and Fourier analysis of ventricular and main arteries pulsations in anesthetized dogs
}

\author{
RAÚL F JIMÉNEZ ${ }^{1}$, PATRICIO TORRES ${ }^{2}$, BRUNO GÜNTHER ${ }^{3}$, ENRIQUE \\ MORGADO $^{4,5}$ and CLAUDIA A JIMÉNEZ ${ }^{6}$
}

\author{
${ }^{1}$ Department of Mathematics, University of Concepción, Chile \\ 2 Department of Surgery, University of Concepción, Chile \\ ${ }^{3}$ Professor Emeritus, Universities of Chile, Concepción and Valparaíso, Chile \\ ${ }^{4}$ Program of Pathophysiology, Faculty of Medical Sciences, University of Santiago, Santiago, Chile \\ ${ }^{5}$ Program of Pathophysiology, Institute of Biomedical Sciences, Faculty of Medicine, University of Chile, \\ Santiago, Chile. \\ ${ }^{6}$ Department of Informatic Engineering and Computer Science, University of Concepción, Chile
}

\begin{abstract}
The purpose of this study was to characterize time-frequency behavior using the Continuous Wavelet Transform (CWT) and Fast Fourier Transform (FFT) to analyze ventricular and arterial pressure signals from anesthetized mongrel dogs. Both ventricular and arterial pressure pulsations were recorded using catheter-tip manometers and the CWT was applied to these signals to obtain module coefficients, associated contours, and the 3-D representation of these modules. FFT was applied to obtain the Fourier spectrum. The mathematical analysis of the cardiovascular pressure pulsations permitted the identification of the evolution of the frequency components for the aortic and pulmonary valve functions as well as the intra-ventricular and respiratory influences on the cardiovascular dynamics. The CWT is a very sensitive and reliable procedure for determining the three-dimensional (time-frequency-amplitude) of the oscillatory phenomena during each cardiac cycle, providing more, although complementary, information than the spectral analysis obtained with the FFT.

Thanks to the FFT, exact values in Hz could be found for the different events produced in each cycle, and thus the information provided by CWT could be related to the information provided by FFT. The combination of both mathematical methodologies permitted identification of each component of the analyzed signals. The $3 \mathrm{D}$ representation allowed an easy comparison of the relative importance of the complex magnitudes in frequency for the different components of the pulsatile waves.
\end{abstract}

Key terms: wavelet transform, Fourier transform, time-frequency-amplitude, ventricular and arterial pressures.

\section{INTRODUCTION}

A signal is the graphical manifestation of a real phenomenon, and it contains information about that real phenomenon that should be recovered and analyzed in order to better understand it.

Scientific and technological development, particularly mathematics and data acquisitions, have allowed the acquisition of more and better information on signals. Indeed, direct observation is no longer sufficient, not even the application of the classic methods based on Fourier's analysis (FFT). Although it is certain that FFT provides precise information about frequency, it does not provide information of possible transient phenomena, nor does it show its evolution.

A new theory was developed in the 1980s in France for the analysis of signals (Grossmann and Morlet, 1985; Grossmann et al., 1989), called the "théorie de ondelettes" or "wavelet theory." This theory provides good, precise information on frequency that is simultaneous and well- 
located in time. Two well-known versions of this theory are the Discrete Wavelet Transform (DWT) and the Continuous Wavelet Transform (CWT).

The applicability of DWT was discussed in Jiménez (1991), and its physiological meaning in Aldroubi and Unser (1996); Günther et al. (1993). Applications of the theory in cardiovascular medicine have recently appeared in Burke and Nasor (2002); Chan et al. (2001); Chen (2002); Gamero et al. (2002); Wiklund et al. (2002). All of these authors have dealt with the cardiac frequency variability or the R-R interval of the ECG.

CWT provides information by means of a three-dimensional representation of timefrequency-amplitude. We have adapted some algorithms that are based on wavelet theory. The sensitiveness and accurateness of CWT have also been discussed in previous papers (Günther and Jiménez, 1996; Jiménez et al., 1997; Jiménez et al., 1999), and for these reasons the same methodology was utilized in the present study on anesthetized dogs. Nevertheless, we have realized that the information provided by CWT is not sufficient, and consequently it is necessary to rely on the information provided by Fourier analysis. In this way, the two methodologies, classic and modern, are complemented, and additional information on cardiovascular physiology can be obtained.

\section{MATERIALS AND METHODS}

The experiments were conducted in mongrel dogs of both sexes, weighing 10$15 \mathrm{~kg}$. Sodium pentobarbital $(30 \mathrm{mg} / \mathrm{kg}$ ) was administered IV as one bolus. The trachea was intubated to assure assistance in breathing $(\approx 12 \mathrm{~b} / \mathrm{m})$. The left ventricular pressure recordings were obtained by means of a micro-tip pressure transducer (model SPG-350, Millar Instruments, USA), which was introduced into the left femoral artery until the left ventricle was reached. For the right ventricle, a similar catheter was introduced into the jugular vein until the catheter tip was located in the right ventricle. For the arterial pressure recordings, another catheter-tip manometer was introduced until the catheter tip reached the aortic valves (juxtavalvular position) and then withdrawn one centimeter. Finally, another arterial catheter was introduced into the pulmonary artery, where the micro-tip was located one centimeter from the pulmonary valves (also with juxtavalvular position and subsequent withdrawal of one centimeter).

The recordings shown in this work correspond to the animal with the greatest regularity of the heart rate.

The present study was carried out in the Experimental Surgical Laboratory of the Faculty of Medicine, University of Concepción, Chile, following the rules of the National Institute of Health Guidelines on Care and Use of Laboratory Animals, USA. Furthermore, these experiments were approved by the Ethics Committee of the Faculty of Medicine and by the Research Board of the University of Concepción, Chile, as well as by the National Commission for Scientific Research of Chile (CONICYT).

\section{Data processing}

Analog signals of pressure oscillations were measured using a data acquisition board Lab-PC 1200 (National Instruments, USA) at a rate of $150 \mathrm{~Hz}$. These data were fed into a personal computer and displayed using the LabVIEW software (National Instruments, Austin, TX USA), and analyzed in the Department of Mathematics of the University of Concepción, Chile.

The CWT yielded a three-dimensional image, a wavelet coefficient module, which represents a surface, where the axes are time, frequency, and amplitude. The third dimension corresponds to the coefficient amplitude (module) and is represented by a color palette where blue-violet is the minimal value and purple-red is the maximal value. The wavelet coefficient values are relative to the signal under study; they are not expressed in physical measure units, but rather provide information regarding the energy quantity contained in the signal (Jiménez et al., 1997). Consequently, the palette color is referred 
to as the relative energy scale. In this study, 20 lines were utilized; i.e. the palette color was divided into 20 categories. In the figures, we present the contour of the module, corresponding to the level curves of the coefficients' surface. The vertical axis, where frequencies are measured, is in a logarithmic scale whose unit is the octave. This scale is related to the Hertz frequency scale provided by Fourier spectrum: octave $=\log$ arithm on base 2 of frequency, i.e., $2^{\mathrm{n}}$ octaves $=\mathrm{n} \mathrm{Hz}$ (Jiménez, 1991).

In the wavelet analysis, the frequency axis should be measured in octaves (such as in a musical scale), and not in Hz. This measure is sub-divided into voices. We have been working with a frequency-band width of 10 or 12 octaves, depending on the requirements of the situation, but always with 8 voices each. Using such values, we obtained an image that provided the 'continuous' of CWT.

The numerical output of CWT application to a signal is a complex matrix of wavelet coefficients, where the sampling points (the horizontal axis represents time) and the total number of voices (vertical axis) provide the dimension. Every complex number can be expressed as a module and a phase, and since in this study a module has an easier graphical interpretation, it was the only one considered. The 3-D module representation demonstrates the relative energy magnitude in the different frequency ranges.

The chosen perspective in this 3-D representation was the one that permitted visualization of very low frequency activity (VLF).

For the calculation of CWT and FFT, it was necessary to have a sample number that was a power of 2. In this case, the contour of the module (Figs B) display 'edge effects' manifested by color change in the curves, an inherent mathematical artifact of the method.

Finally, the Fourier spectrum was calculated considering a number of harmonics within a scale frequency from 0 to $22 \mathrm{~Hz}$ for the left ventricle and 0 to $16 \mathrm{~Hz}$ for the right ventricle, from 0 to $20 \mathrm{~Hz}$ for pulmonary artery, and from 0 to $42 \mathrm{~Hz}$ for the aorta. The frequencies beyond this range were considered irrelevant for the study.
RESULTS

\section{Time frequency analysis (TFA) of left ventricle}

Figure 1A displays the 16 pulses of left ventricular (LV) pressure, lasting 6.82 seconds, and registered at a rate of $150 \mathrm{~s} / \mathrm{s}$. It is observed that diastolic pressure remains practically constant; nevertheless, systolic pressure displays one slight variation that corresponds to the influence of the assisted breathing. It is possible to observe that during the first third of ventricular ejection, a peak appears in each pulse that corresponds to the maximal ventricular pressure (MVP). This phenomenon can be seen with greater clarity in Figure 1E.

Figure 1B presents the contour of the CWT-provided module of the same 16 pulses shown in Fig 1A. We can observe that all frequency activity is between 0 and 4.5 octaves, i.e., between 1 and 22.6 Hertz. The color palette has a relative variation between 10 and 150 units.

It is possible to observe an intense band of red between 1 and 1.5 octaves, indicating that it constitutes the greatest energy event and that it corresponds to heart rate frequency (HR). FFT assigns this event a value of 2,344 Hz. In the range between 1.5 and 4.5 octaves, a series of lower-energy events appeared in different intensities of blue. In order to study these events more accurately, the 4 pulses of Figure 1 indicated by letter $\mathrm{E}$ were plotted in Figure $1 \mathrm{E}$. Figure 1F displays the module contour of the same pulses.

Figure $1 \mathrm{C}$ presents the three-dimensional image of the contour with a perspective chosen to show the activity of VLF. In this graph, HR is seen as the tallest peak, and it displays a small undulation corresponding to an amplitude modulation (AM) phenomenon related to breathing. The small peak to the left of HR corresponds to ventricular ejection (VE), and the one to the right corresponds to the maximal ventricular pressure (MVP). In the low frequency zone, small undulations corresponding to the respiratory influence (RI) can be observed. 
FIGURE 1. Wavelet and Fourier analysis of left ventricular pressures (LVP)

A) Original tracing of pressure pulsations

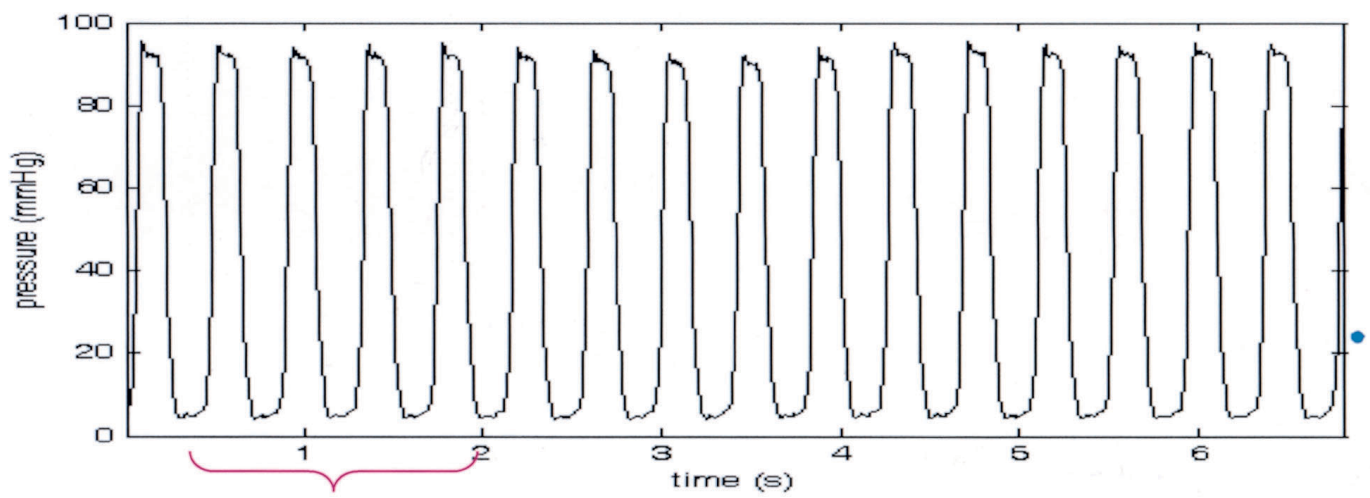

(E)

B) Contour module

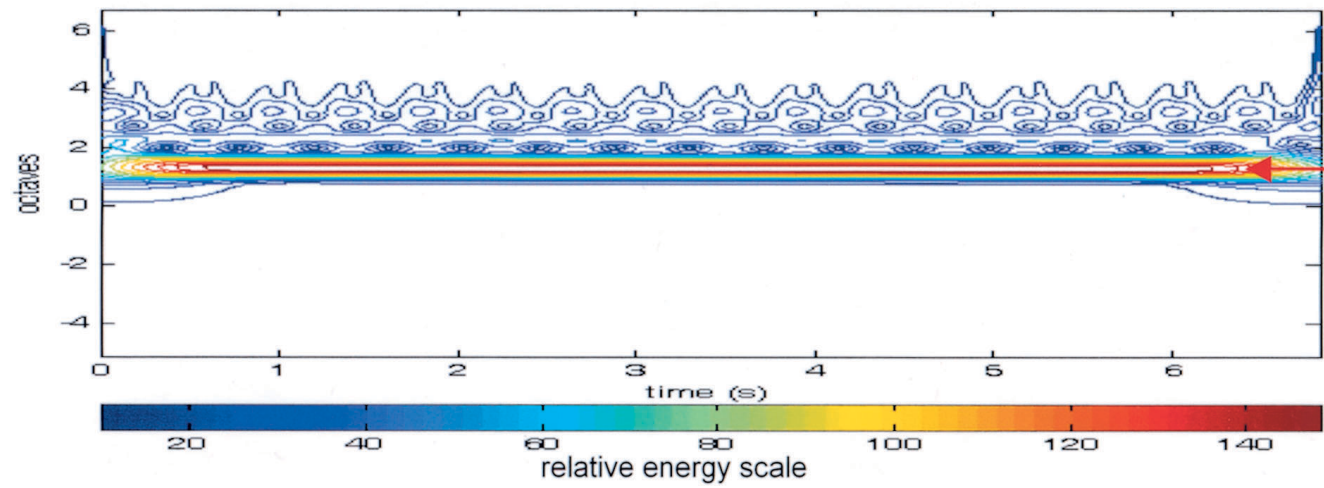

C) 3D module

D) Fourier spectrum
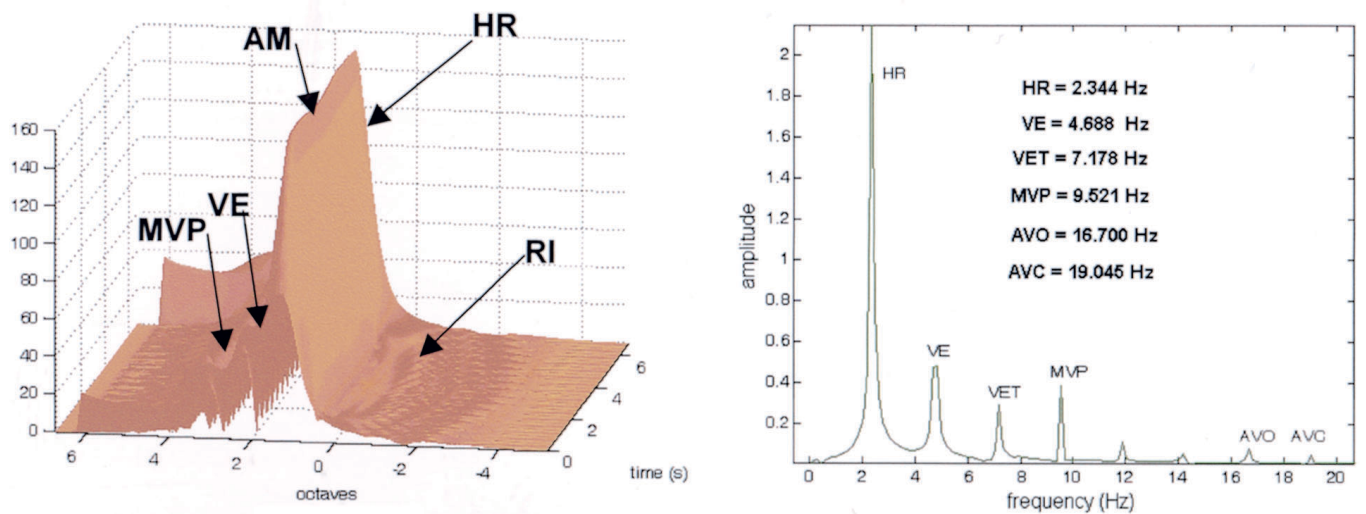
E) Original tracing of pressure pulsations (labeled as E in Fig $1 \mathrm{~A}$ )

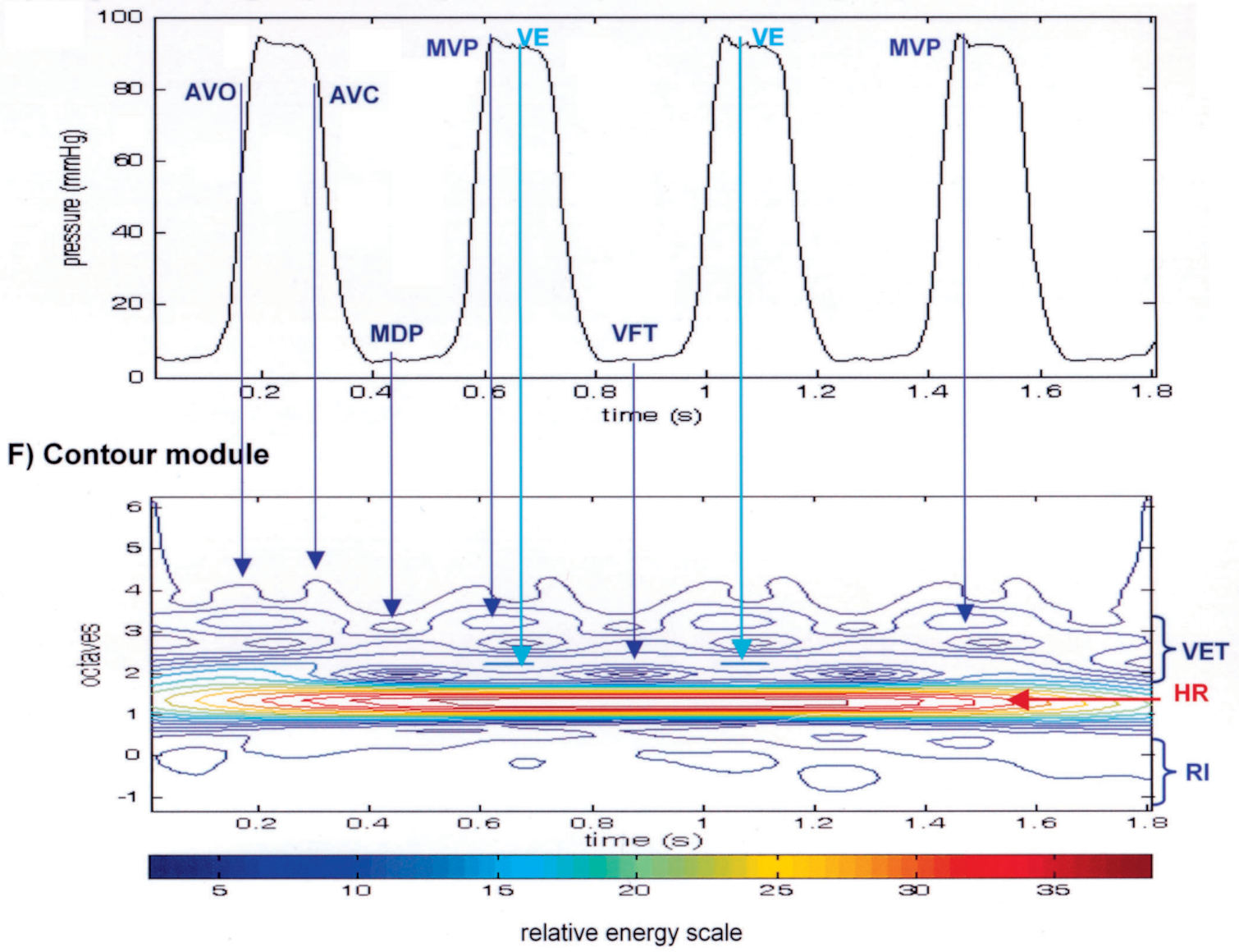

Figure 1:

- A: 16 pulses of left ventricular (LV) pressure, lasting 6.82 seconds and registered at a rate of $150 \mathrm{~s} / \mathrm{s}$. The first four pulsations (E) are amplified in Figure 1E.

- B: Contour of the module given by CWT of the pulses shown in Fig. 1A.

- C: Three-dimensional contour image. HR, ventricular ejection (VE), maximal ventricular pressures (MVP) and respiratory influence (RI).

- D: Fourier spectrum of the original recordings.

- E: The first four pulsations from Figure 1A, shown in an expanded time scale.

- F: Contour module of the pressure pulsations shown in Figure 1E.

Figure 1D shows the Fourier spectrum. Events greater than $21 \mathrm{~Hz}$ do not appear because they are of an insignificant magnitude.

In Figure 1E, the MVP can be observed more clearly. It is also possible to observe small disturbances during the ventricular ejection that they probably correspond to turbulences caused by the exit of the blood as well as very small turbulences during ventricular filling (VFT).

In Figure $1 \mathrm{~F}$, the module contour is shown on a scale from -1 to 6 octaves. It is interesting to note that all the activity is between -1 and 4 octaves, i.e., between 0.5 and 16 Hertz. When analyzing this contour, we can visualize and identify, in addition to HR, the following events:

\section{Ventricular ejection (VE)}

VE follows $\mathrm{HR}$ in relative energy. The series of dashed lines that appear in turquoise at a level of 2.3 octaves was associated to VE. According FFT, it has a value of 4,688 Hertz. 


\section{Ventricular ejection turbulences (VET)}

Above the VE line, another band appears approximately between 2.8 and 3.8 octaves, formed by several complexes of blue color (low energy) that can be associated with the turbulence produced during VE. From the chosen perspective, this event is not seen in C. FFT detects this activity as 3 complexes: the first of medium amplitude with a value of $7,178 \mathrm{Hertz}$, the other two are of very low amplitude and with values around 12 and 14 Hertz, respectively.

\section{Ventricular Maximal Pressure (MVP)}

CWT detects the small peak produced during the first third of the ventricular ejection (VE). A peak appears that corresponds to the maximal ventricular pressure, as a series of light blue oval-shaped curves before 3.3 octaves. According to FFT, it has a value of $9.521 \mathrm{~Hz}$.

\section{Ventricular filling turbulences (VFT)}

CWT detects the turbulences produced by the ventricular filling as very low energy complexes, expressed as blue-indigo concentric curves around 2 octaves. CWT also detects another small event around 3.1 octaves, like small blue ovals, marked MDP (maximal diastolic pressures) and that correspond to a small pressure increase during the ventricular filling, almost imperceptible in the original record. FFT does not detect any of these phenomena.

\section{Aortic valve opening (AVO) and Aortic valve closure (AVC)}

In the original record (Figure 1E), it is not possible to see aortic valve opening or closing. However, CWT detects these phenomena in the form of a series of blue two-phase curves between 3.6 and 4.4 octaves, approximately. The first peak corresponds to an imperceptible change in the shape of the pressure curve produced immediately after aortic valve opening (AVO); FFT detected it with a value of $16.700 \mathrm{~Hz}$, a very small amplitude. The second peak corresponds to a change in the wave shape produced immediately after the aortic valve closing (AVC) with a value of $19.045 \mathrm{~Hz}$ provided by the FFT, and with even smaller amplitude. These events are not visible in the 3-D image, due to the chosen perspective and their negligible magnitude.

\section{Respiratory Influence (RI)}

The breathing influence observed in the original recordings is visible in Figure $1 \mathrm{~F}$ as very low energy activity between -1 and 0 octaves. It can also be observed clearly in the 3-D image.

\section{TFA of the right ventricle}

Figure 2A shows sixteen pulsations of right ventricular (RV) pressures during 6.82 seconds. The maximal systolic pressures remain practically constant, whereas the diastolic pressures present slight variations (2 $\mathrm{mmHg}$ ) that correspond to the influence of the assisted breathing. It can also be observed that the systolic pulsations are triangular in shape, which are different than those of the left ventricle, which are almost rectangular.

The contour of the module provided by CWT of the pulses are presented in Figure 2B. As in the left ventricle, it can be observed that the entire frequency activity is between 1 and 4 octaves. In this case, the color palette has a relative variation from 1 to 25 units. The behavior in timefrequency-amplitude of the right ventricle is similar to that of the LV, except in the zone of high frequency around 4 octaves, which is not biphasic.

Figure 2C presents the 3-D contour image of the right ventricle. The events are very similar to those of the LV.

Figure 2D presents the corresponding Fourier spectrum. The frequency characteristics are similar to those of LV.

In order to more accurately visualize the low and high frequency events, 4 pulses of the original record, marked with the letter 'E' are plotted in Figure 2E. Small disturbances can be observed during VE that probably correspond to turbulences caused by blood exiting (VET). It is also possible to observe small amounts of turbulence during the ventricular filling (VFT). 
FIGURE 2. Wavelet and Fourier analysis of right ventricular pressures (RVP).

A) Original tracing of pressure pulsations

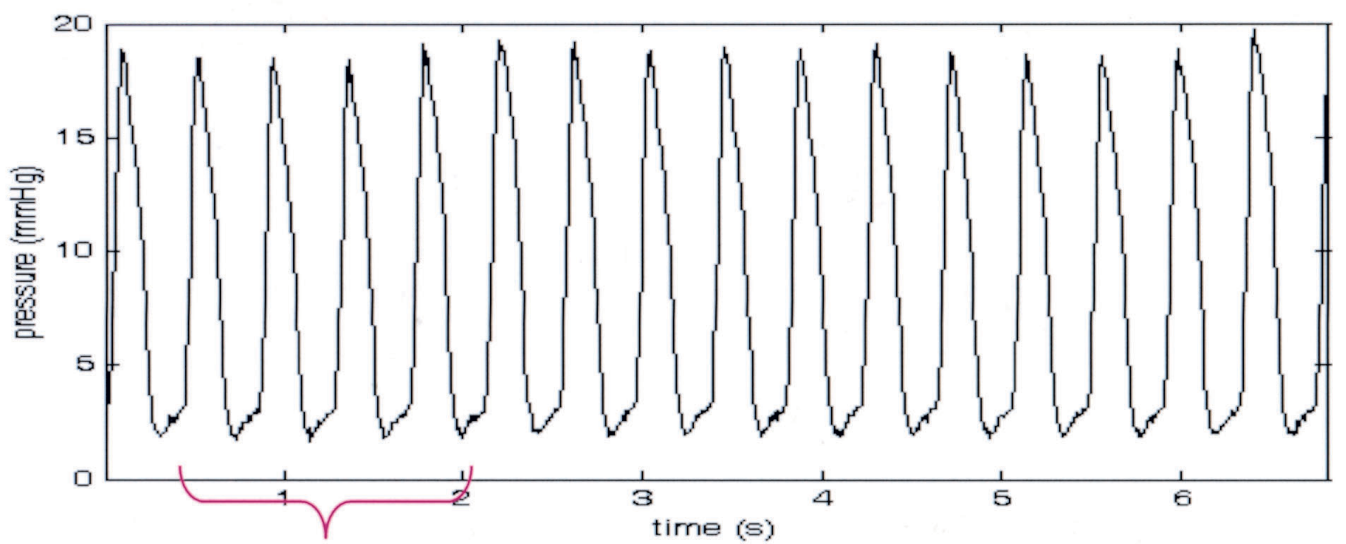

(E)

B) Contour module

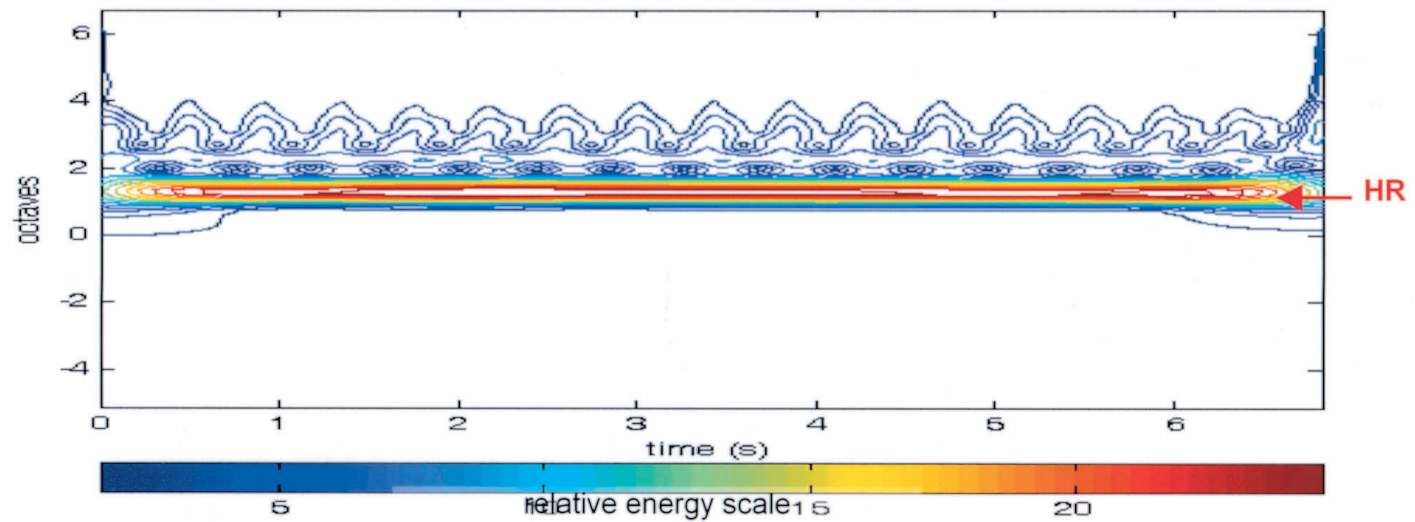

C) 3D module

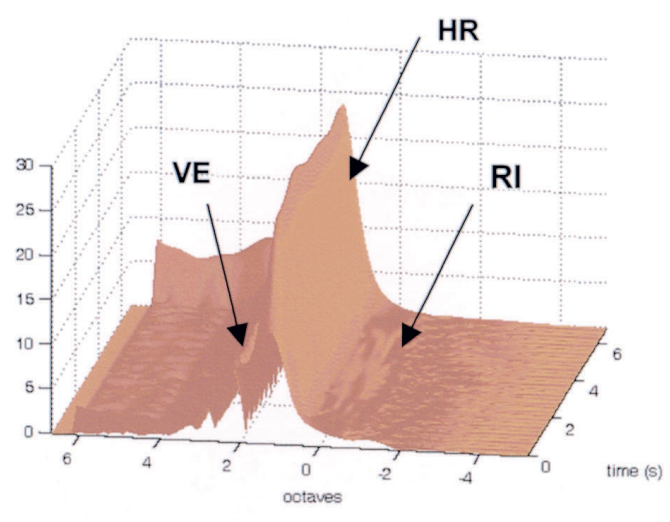

D) Fourier spectrum

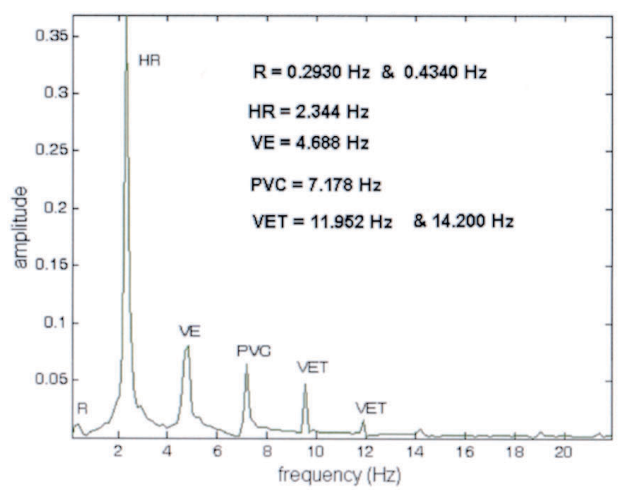


E) Original tracing of pressure pulsations (labeled as E in Fig $2 \mathrm{~A}$ )

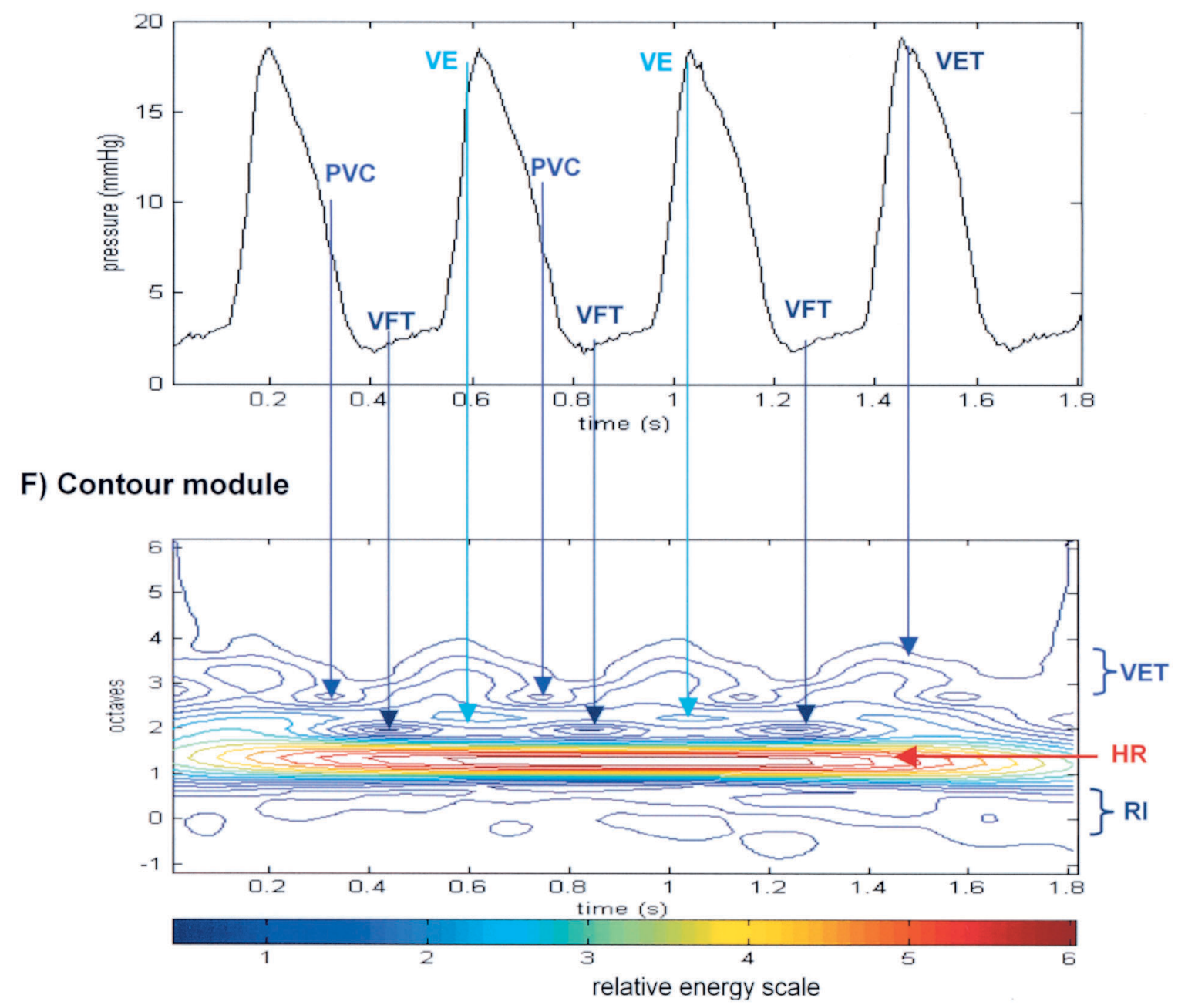

Figure 2:

- A: 16 pulses of right ventricular (RV) pressure. The first four pulsations (E) are amplified in Figure 2E.

- B: Contour of the module given of the same pulses shown in Figure 2A.

- C: Three-dimensional contour image.

- D: Fourier spectrum of the original recordings.

- E: First four pulsations from Figure 2A, shown in an expanded time scale.

- F: Contour module of the 4 pressure pulsations from Figure 2E.

In Figure $2 \mathrm{~F}$, the module contour in a scale from -1 to 6 octaves of these 4 pulses is shown. The TFA of the RV is very similar to the $\mathrm{LV}$, with the exception that:

- VE does not present a pure frequency in this case and is depicted as a closed curve rather than a line, although both have the same value $(4.688 \mathrm{~Hz})$.

- The valve opening is not detected in this case, and the closing takes place with a smaller frequency of $7.178 \mathrm{~Hz}$.

- The Fourier spectrum (Fig. 2D) detects the influence of breathing with a value of $0.360 \mathrm{~Hz}$. 


\section{TFA of the Aortic Blood Pressure}

In Figure 3A, the original records of 15 pulses of aortic pressure registered during 6.83 seconds, $1 \mathrm{~cm}$ of the valves are shown. The influence of assisted breathing can also be observed in both systolic and diastolic pressures.

In Figure 3B, the module contour of the records is shown. All the significant activity is between -3 and 6 octaves. HR appears in red between 1 and 1.5 octaves, similar to the ventricular record, although the FFT value is $2.197 \mathrm{~Hz}$ in this case. Above the HR, another orange-yellow band appears between 2 and 2.5 octaves that corresponds to ventricular ejection (VE) with an FFT value of $4.541 \mathrm{~Hz}$. In the range limited by 2.5 and 6 octaves, a series of unidentified low energy events appears. Finally, around -2 octaves, very low energy activity corresponding to respiratory influence (RI) can be observed.

Figure $3 \mathrm{C}$ shows the 3 -D contour image. HR appears as the highest peak, and we can observe an undulation in the upper part that corresponds to the previously-mentioned AM-phenomenon. VE is observed as one peak of medium altitude located to the left of the peak corresponding to the HR. Furthermore, two more small peaks can be visualized that correspond to the $\mathrm{AVO}$ and the dichrotic notch (DN), respectively. RI is visible in the low frequency zone.

The spectrum given by FFT is shown in Figure 3D with a wide band (wb): 0 to $41 \mathrm{~Hz}$.

To visualize and identify the CWT events and relate them to the phenomena of the original records, Figure $3 \mathrm{E}$ presents the first 4 pulses of the original record, and the respective contour is depicted in Figure 3F.

In Figure 3E, the dichrotic notch (DN) and diastolic turbulences can be clearly observed. In diastole time, it is possible to identify two phases: the first begins immediately after DN, where a slow pressure drop $\left(\mathrm{F}_{1}\right.$, protodiastolic) takes place, and a second phase where the pressure drops more quickly $\left(\mathrm{F}_{2}\right.$, telediastolic).

Figure $3 \mathrm{~F}$ shows the module contour. Several phenomena can be identified in this figure:
- Diastolic waveform changes (Ch): Above the yellow-orange band (VE), and around 2.8 octaves, a series of blue spots appear. These correspond to the slope change of the pressure curve during the diastolic phase $\left(F_{1}\right.$ to $\left.F_{2}\right)$. The FFT value of this phenomenon is 6.738 $\mathrm{Hz}$, a major amplitude (3rd location).

- Aortic valve opens (AVO): The opening of the valves is expressed by small closed curves in turquoise (medium energy) around 3.8 octaves, where the FFT value of this event is 13.477 Hz. In the 3-D image, this phenomenon is visible as the third peak to the left of the HR peaks.

- Turbulences (T1 and T2): Blue curves can be seen between 3 and 5 octaves. They can be associated with turbulences (T1 and T2) during the systolic, diastolic and valve closing phases. Fourier detects these activities with values of 8.935 , 11.135 (T1), and $15.674 \mathrm{~Hz}$, and one wb of 31.048 to $38.086 \mathrm{~Hz}$ (T2).

- End systolic turbulences (EST): Between 4.3 and 4.9 octaves, small events are observed in blue. They correspond to turbulences during the final systolic phase. The FFT values of these activities are 20.068 and 29.150 $\mathrm{Hz}$, respectively, with very small amplitude.

- Aortic valve closes (DN): The noticeable dichrotism observable in the original records is clearly expressed in the high frequency zone, between 5 and 6 octaves, as complexes in turquoise blue (DN), with a medium relative energy. The FFT value of these complexes is $40.285 \mathrm{~Hz}$, with very low amplitude.

- Respiration (R): the influence of breathing is expressed as blue-colored curves between -1 and -2 octaves, with an FFT value of 0.293 Hertz.

\section{TFA of Pulmonary artery}

In Figure 4A, the original records of 15 pressure pulses during 6.83 seconds registered in the pulmonary artery are shown. The influences of the assisted breathing can be clearly observed. 
FIGURE 3. Wavelet and Fourier analysis of aortic pressures.

A) Original tracing of aortic pressure pulsations

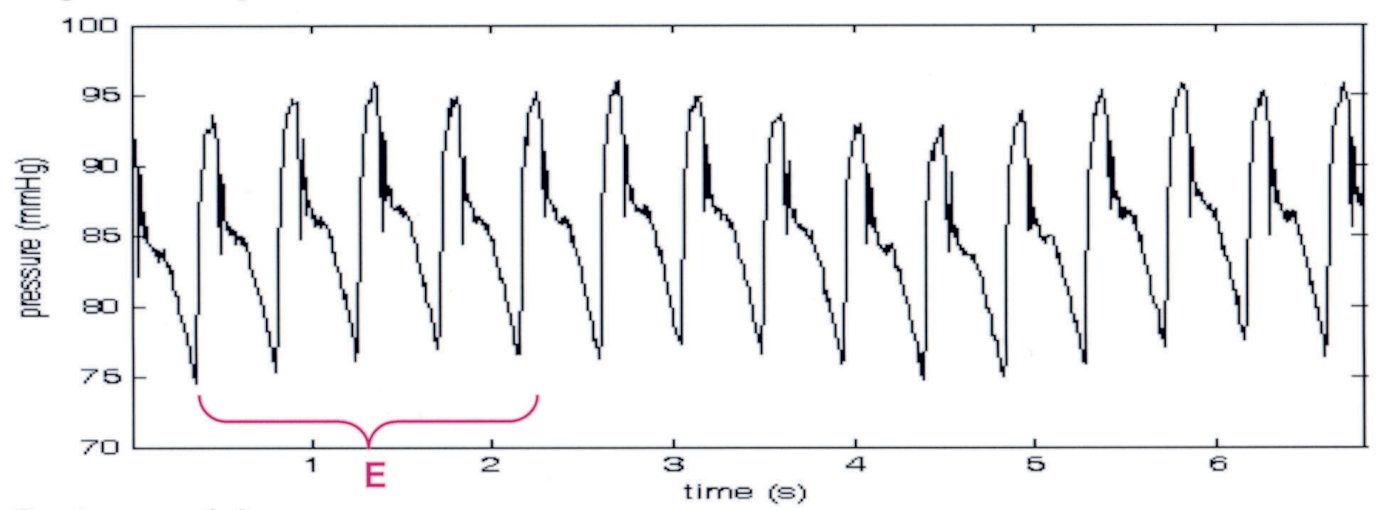

B) Contour module

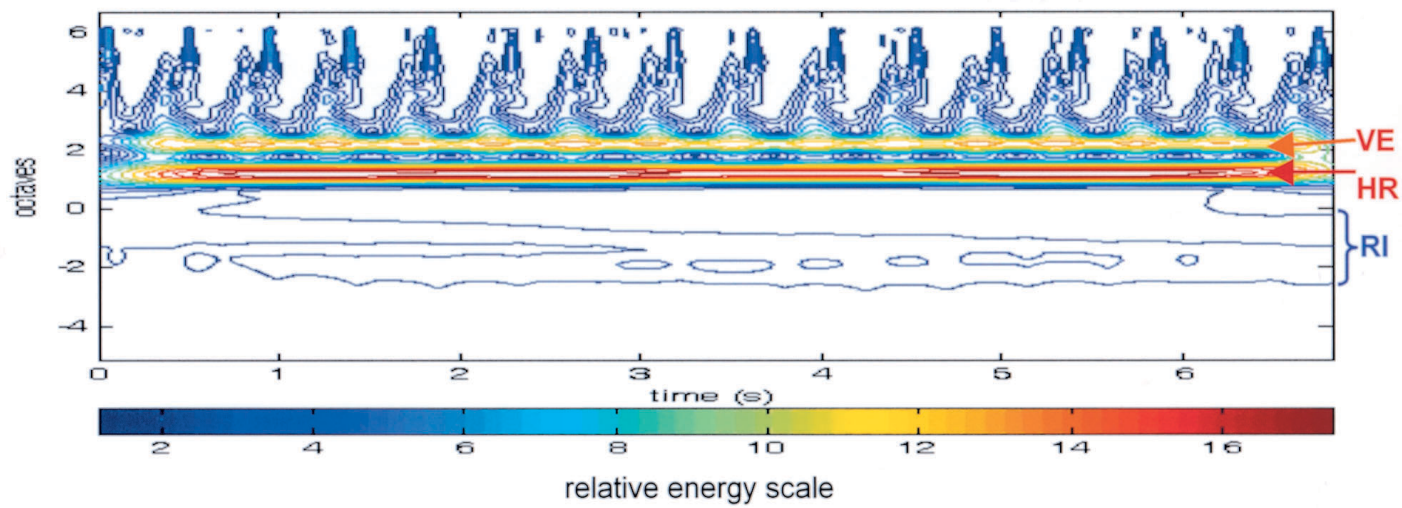

C) 3D module

D) Fourier spectrum
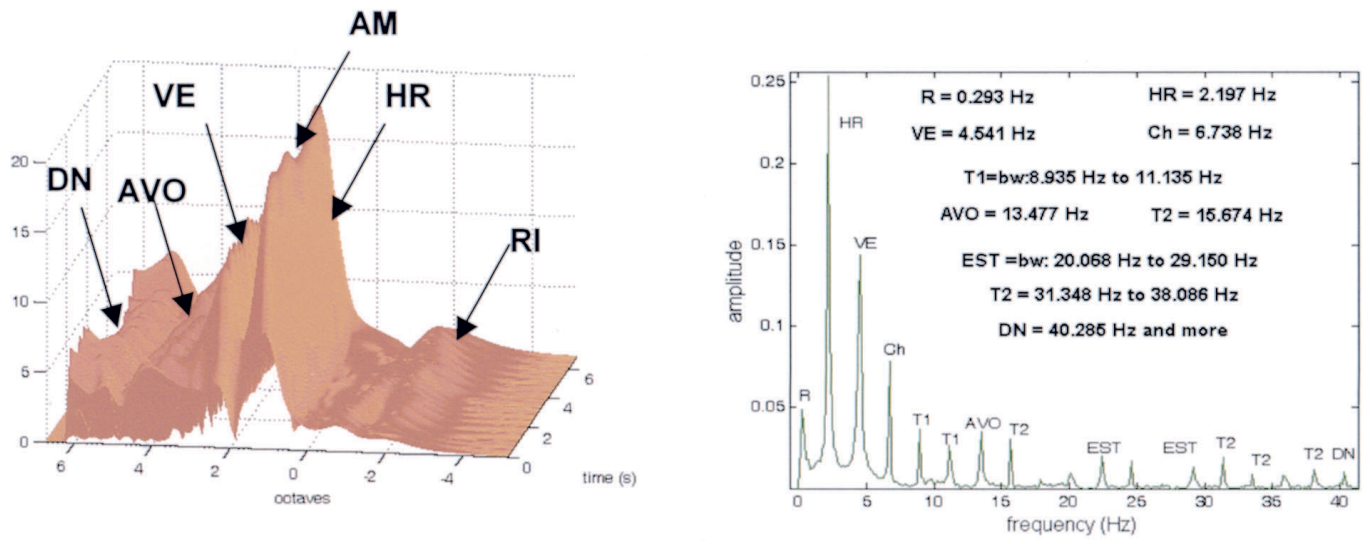
E) Four pulses of aortic pressure pulsations (labeled as E in Fig $3 \mathrm{~A}$ )

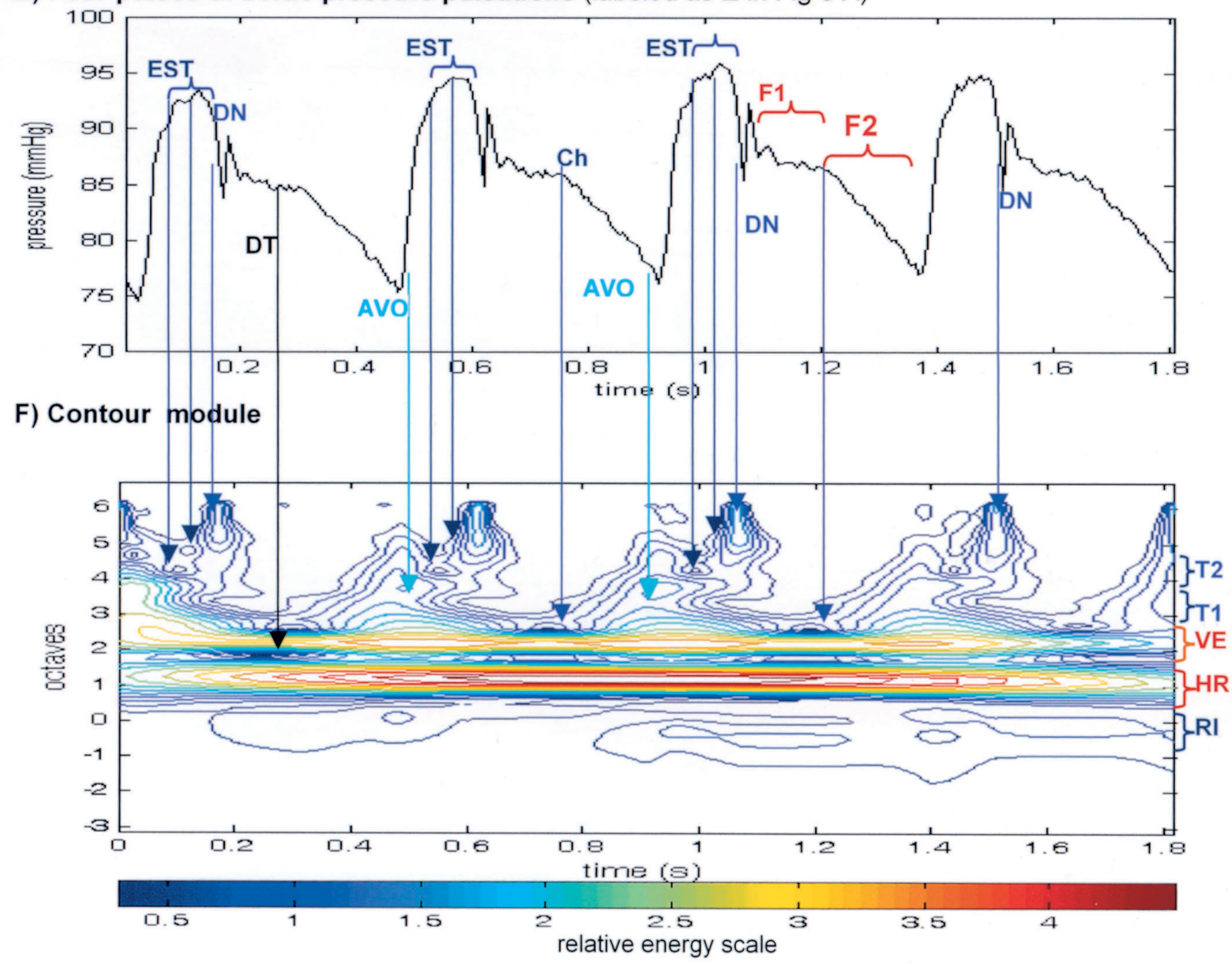

Figure 3:

- A: Original records of 15 pulses of aortic pressure registered over 6.83 seconds.

- B: Contour module of these records.

- C: Three-dimensional contour image.

- D: Fourier spectrum of the original recordings.

- E: First four pulsations from Figure 3A, shown in an expanded time scale.

- F: Contour module of the 4 pressure pulsations from Figure 3E.

In Figure 4B, the module contour of the same registry is shown. The TFA in the pulmonary artery is similar to the one in the aorta, but in this case the dichrotic notch $(\mathrm{DN})$ is less marked. The RI is more notable in this case. Between 2.5 and 6 octaves, a series of events of low energy can be observed, but they cannot be identified in this figure.

In Figure $4 \mathrm{C}$ it is possible to observe more clearly the AM phenomenon in the HR peak, the ventricular ejection (VE), pulmonary valve opening (PVO) and the respiratory influences $(\mathrm{RI})$.
In Figure 4D, the Fourier spectrum is presented. It has a smaller wb than in the aorta and the frequencies over $20 \mathrm{Hertz}$ are negligible.

To better visualize and identify the phenomena of the original records, the first 4 pulses of the recordings are shown in Figure 4E, and its contour in Figure 4F. In this figure other phenomena can also be identified:

- Change 1 (Ch1): Around 1.9 octaves, CWT detected a series of blue spots that corresponds to the slope change of the pressure curve at the end of DN. 
FIGURE 4. Wavelet and Fourier analysis of arterial pulmonary pressures.

\section{A) Original tracing of pulmonary pressure pulsations}

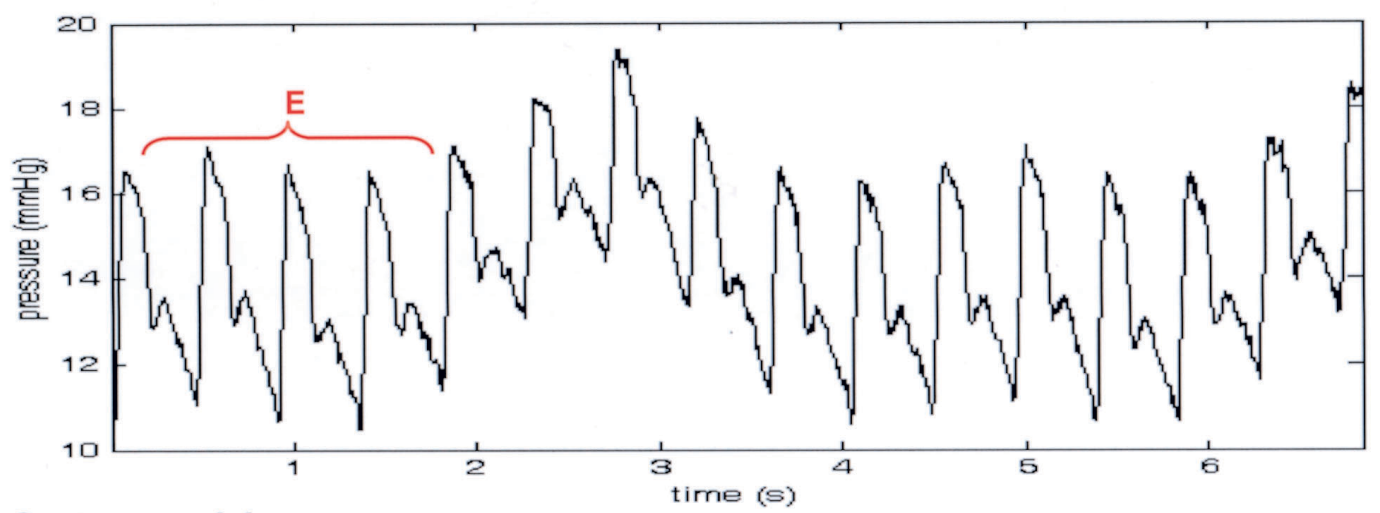

B) Contour module

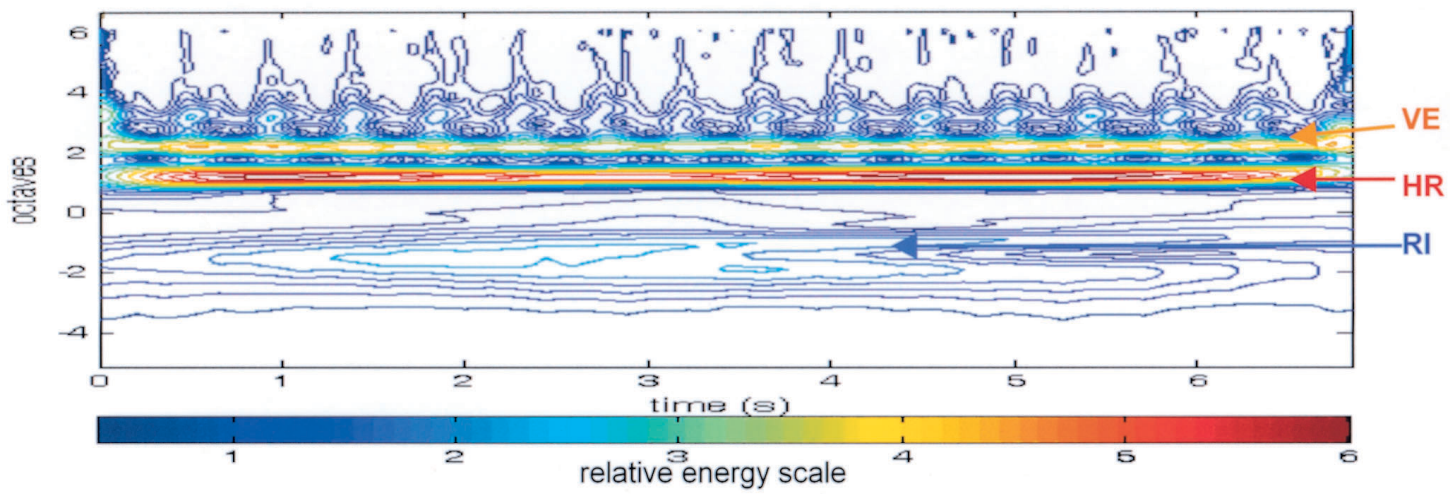

C) 3D module

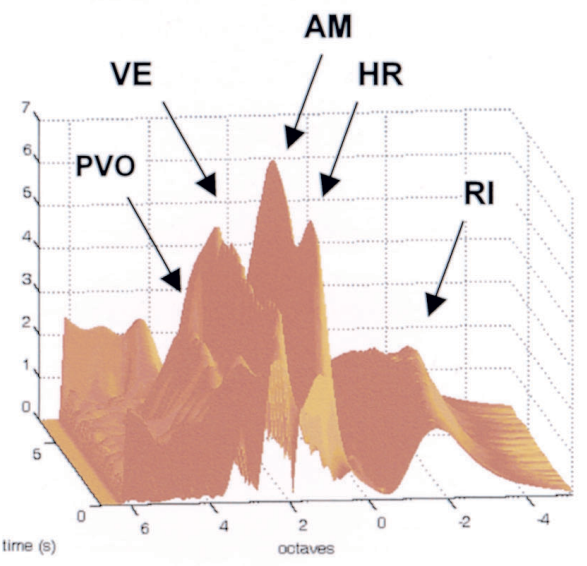

D) Fourier spectrum

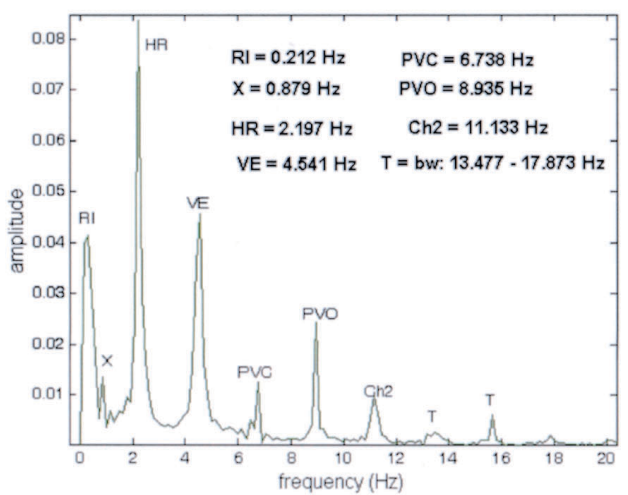


E) Original tracing of four pulmonary pressure pulsations (labeled as $E$ in Fig 4A)

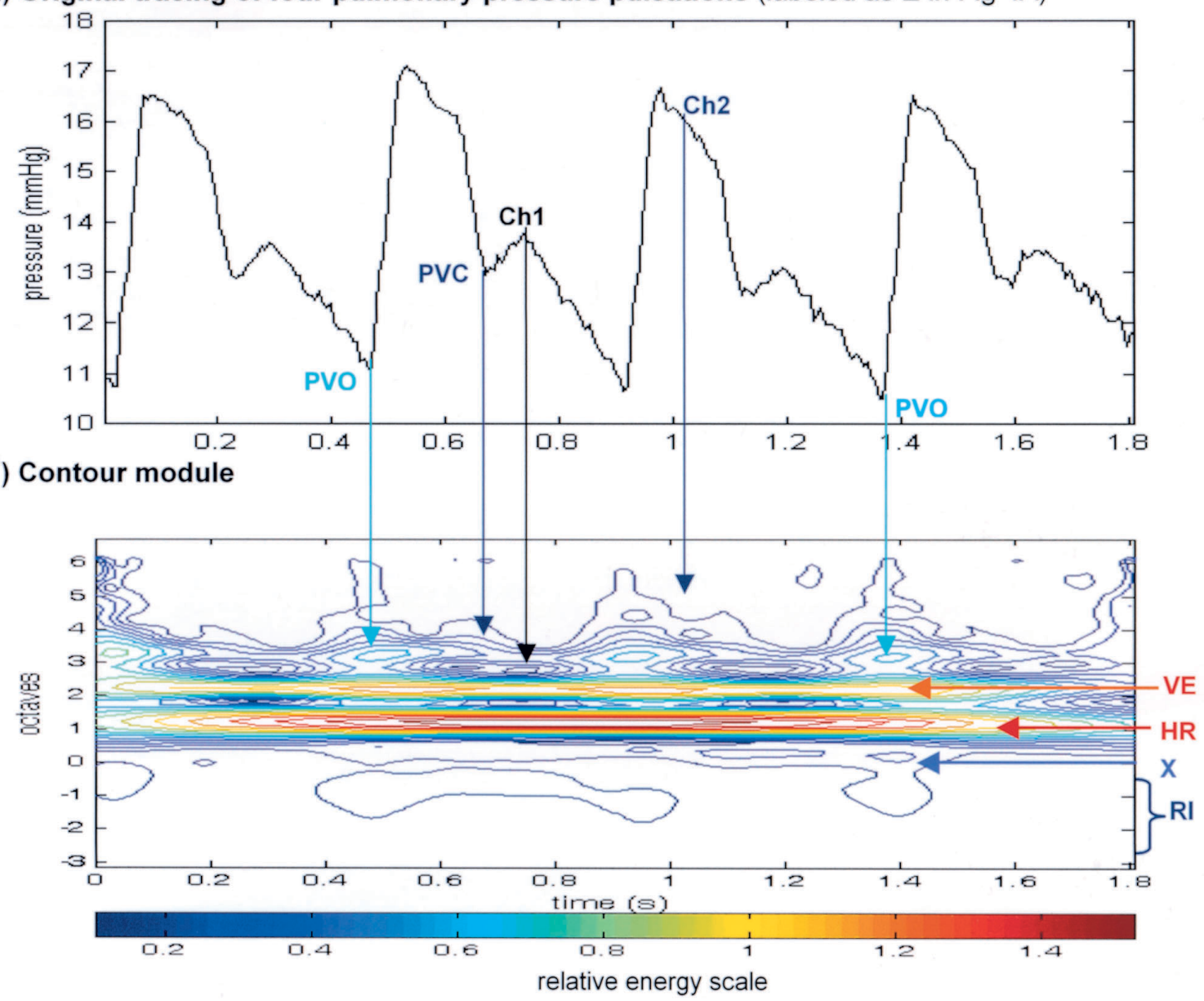

Figure 4:

- A: Original records of 15 pulses of pulmonary pressure registered over 6.83 seconds. The first four pulsations (E) are amplified in Figure 4E.

- B: Contour module of these records.

- C: Three-dimensional contour image.

- D: Fourier spectrum of the original recordings.

- E: First four pulsations from Figure 4A, shown in an expanded time scale.

- F: Contour module of the 4 pressure pulsations shown in Figure 3E.

- Pulmonary valve closing (PVC): The pulmonary valve closing is shown in this case as blue-colored curves around 2.8 octaves. FFT assigns this phenomenon a frequency of $6.738 \mathrm{~Hz}$ with a small magnitude.

- Pulmonary valve opening (PVO): The sigmoid valve opening is expressed by small, turquoise-colored closed curves around 3.4 octaves that occur in a lower frequency $(8.935 \mathrm{~Hz})$ than in the aorta.

- Change 2 (Ch2): Around 3.8 octaves, CWT detects a change in the shape of the wave during the systolic period that follows the maximal systolic pressure (Ch2), viewed as small circles of very low energy (blue). FFT assigns this phenomenon a value of $11.133 \mathrm{~Hz}$ with small amplitude.

- Turbulences (T): Between 4 and 6 octaves, it is possible to observe activity with very high frequency but very low energy, and that corresponds to the turbulences produced during the systolic and diastolic phase. FFT assigns to this phenomenon a wide band of 13.477 to $17.873 \mathrm{~Hz}$. For. 
- Respiratory Influence (RI): RI is detected between -1 and -2 octaves with a FFT value of $0.212 \mathrm{~Hz}$.

- X Phenomenon (X): Between 0 and -1 octaves, CWT detects a very low energy activity with an FFT value of 0.879 Hertz. Its origin could not be identified in this graph.

With the aim of identifying the $\mathrm{X}$ phenomenon, Figure 5A shows an extended original record of the pulmonary pressures with 1500 samples equivalent to 10 seconds. In this figure, we can see the influence of a complete respiratory cycle $(\mathrm{R})$, and the effects of inspiration (i) and expiration (e), respectively.

In Figure 5B, the influence of assisted breathing (RI) is shown; appearing around -2 octaves with an activity of medium energy (in green). Phenomenon $\mathrm{X}$ appears around -1 octave as an activity of smaller energy (in blue) that corresponds to the influence of the inspiratory or expiratory movement of the assisted breathing. This phenomenon can also be visualized in the 3-D image as a bifurcation that begins to the left of the RI peak.

\section{DISCUSSION}

The shapes of the pressure waves obtained in this study are not similar to those classically described in the literature (Best and Taylor, 1985; Fung, 1984; Günther and Jiménez, 1996). These differences can be explained by taking in account that the classic recordings were made utilizing catheters filled with physiological solutions that present hysteresis and that they were made at sampling frequencies between 10 and 15 Hz. In our work, we used highly sensitive electronic catheters, with no hysteresis, and the sampling frequency was 150 Hertz, which permits a more accurate description of the phenomenon (Jiménez et al., 1999).

Comparing the shape of the pressure pulsations within both ventricles, it can be observed that in the left ventricle the rhythmic systolic events are symmetrical and almost rectangular in shape, while these systolic pulsations are triangular in the right ventricle. These differences are probably due to the fact that the left ventricle is mainly a high-pressure pump, while the right one should be considered primarily as a low-pressure volume pump.

In relation to the behavior in TFA, it is interesting to note that in both ventricles $\mathrm{VE}$ has the same frequency value, despite the differences in wave shape, pressure regime, and relative energy contained in the signal (palette of colors).

It is necessary to emphasize that the VE has a frequency value exactly twice the frequency value of HR, thereby constituting a harmonic phenomenon that is presented as a pure frequency (musical tone) in the left ventricle. In the right ventricle, although FFT assigns the same value, CWT does not identify it as a pure frequency (near tone). It must be remembered that all the frequency values analyzed are below the audible range. An identical behavior is also shown in both cardiac cavities during the ventricular filling (VE). We do not have a hypothesis to explain this result.

The behavior of TFA is slightly different in both ventricles starting in 2.5 octaves (5.6 Hertz). It is remarkable that in the left ventricle a biphasic behavior is demonstrated that accounts for AVO and the AVC. This behavior is not observed in the right ventricle, probably due to the abrupt diminution of the intra-ventricular pressure, possibly due to valve opening.

In the original record, the respiratory influence (RI) is not observed in the LV, and is only be slightly visible in the RV record. By means of CWT, the RI can be visualized in the 3-D image of the module in both ventricles as well as in the contour of the amplified module (Figs. $1 \mathrm{~F}$ and $2 \mathrm{~F}$ ). Interestingly, FFT does not detect this event in the left ventricle, and in the right ventricle it assigns a value of $0.360 \mathrm{~Hz}$ (Fig. 2D). Unlike the left side, RI is most notable in the right ventricle; this phenomenon can be explained by the increase in resistance in the pulmonary circulation when the mechanical respirator makes a positive pressure insufflation and due to the low pulmonary circulation pressure (Günther and Jiménez, 1996). 
FIGURE 5. Respiration effect on arterial pulmonary pressures

A)Original tracing and respiration influences

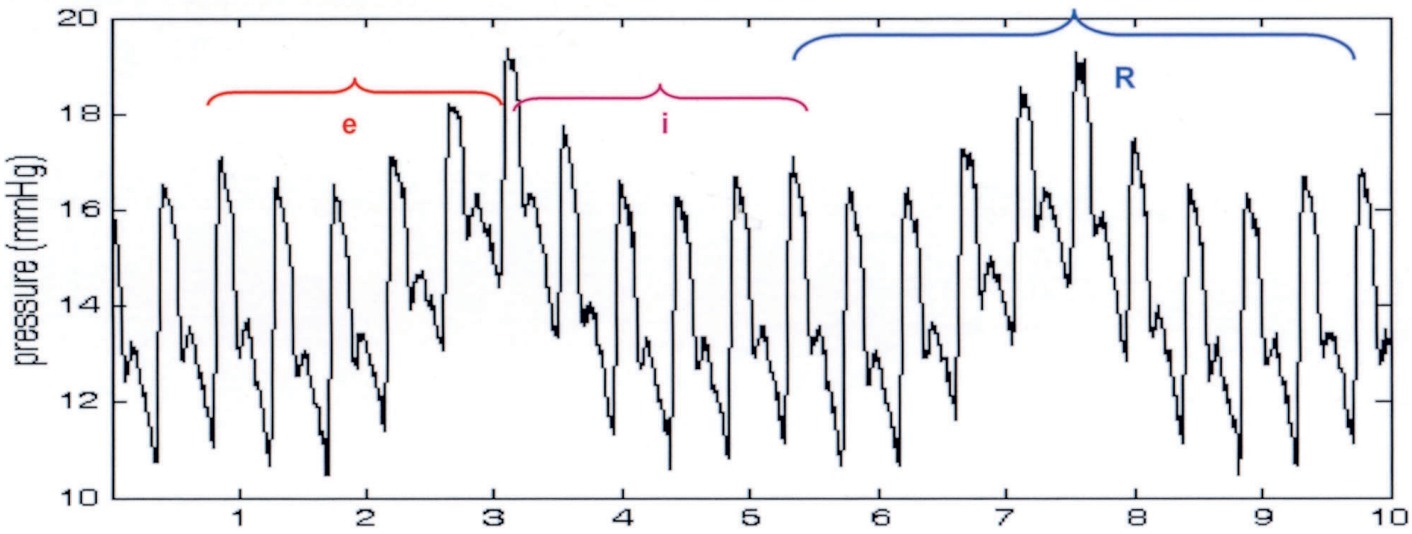

B) Contour module

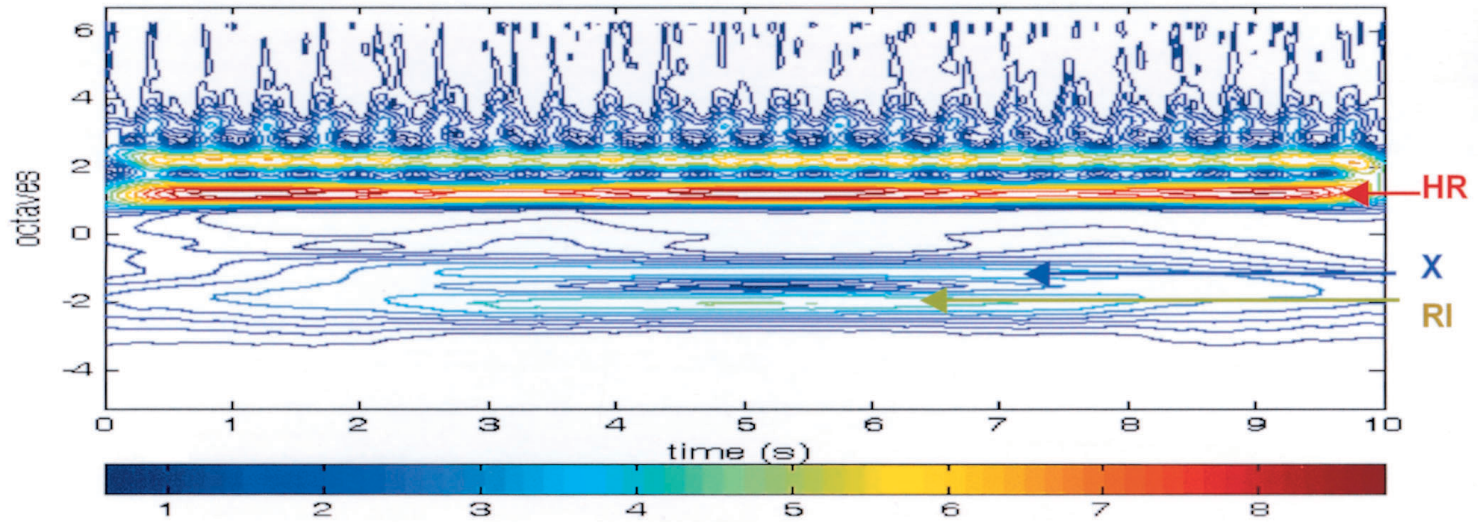

C) $3 \mathrm{D}$ module

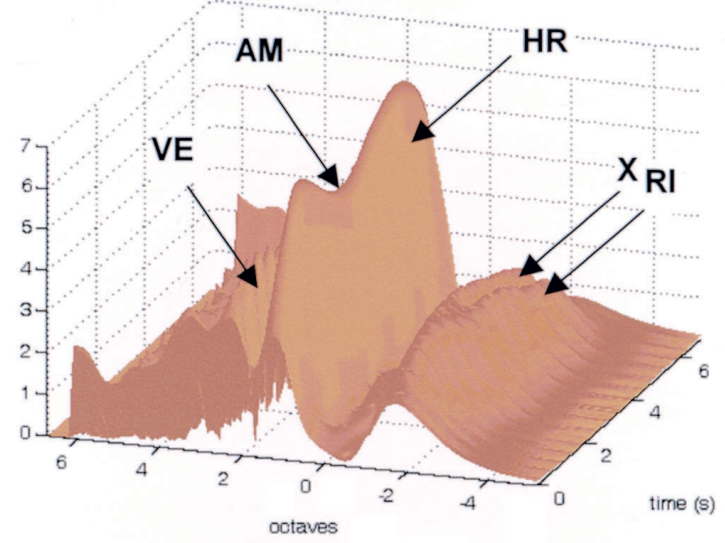

Figure 5:

- A: Original records of pulmonary pressure registered over 10 seconds.

- B: Contour module of the same pressure pulsations from Figure 5A.

- C: Three-dimensional contour image. 
The similar time frequency behavior of both ventricles is remarkable, considering the significant anatomical and physiological differences. This finding reinforces the idea that the two ventricles, despite their differences, are identical in the way they work like a pump (Best and Taylor, 1985; Mohrman and Heller, 1981).

An interesting aspect is that VE in both ventricles is a harmonic of the HR. However, in the arteries, this value is not exactly twice the HR, although very close. We do not have a hypothesis to explain this difference.

Other references concerning the analysis of TFA of the intra ventricular pressure could not be found in the literature.

The dichrotism produced by valve closing is different in the two arteries. In the aorta, it is of very short duration, which generates a complex of high frequency. In the pulmonary artery, however, this dichrotism is slower and causes a smaller frequency complex. This fact can be due to the differences in resistance that each ventricle must surpass (Best and Taylor, 1985; Fung, 1984; Mohrman and Heller, 1981). Additional references concerning the records of arterial pressure at comparable sampling frequencies could not be found in the literature.

The behavior in TFA is similar in both arteries, with the exception of dichrotism, $\mathrm{RI}$ and the AM phenomenon. Indeed, $\mathrm{DN}$ is much more notable in the aorta than in the pulmonary artery, despite its low energy. This is due to the fact that CWT can efficiently detect high frequency transients (Jiménez et al., 1997). Another difference is in the breathing influence; the RI of the aorta is low energy (blue color), while in the pulmonary artery, this influence is more notable and of higher energy (turquoise color). It is interesting to note that in the pulmonary artery, the X-phenomenon also appears and corresponds to the influence of the inspiration or the expiration in the pulmonary artery pressure. These differences could also be explained by the effect of the positive pressure breathing, and by the pressure differences in both arteries (Best and Taylor, 1985; Fung, 1984; Mohrman and Heller, 1981). A final aspect is that the AM-phenomenon has been already described in the aorta (Günther et al., 1993; Günther et al., 1996; Jiménez et al., 2001), which is more evident in the pulmonary artery. This fact reinforces the hypothesis that the modulated signal (MS) of this phenomenon is strongly influenced by the respiratory movements (Jiménez et al, 2001).

\section{CONCLUSIONS}

The results obtained here allow us to conclude that both mathematical methodologies, CWT and FFT, are perfectly complemented by the study of the behavior in TFA of the ventricular and arterial pressure waves. Indeed, CWT is effective in allowing the visualization of:

- the frequency behavior of the different components of the wave pulse

- transient phenomena undetected by FFT

- the relative energy value contained in the signal

- any change in the evolution of the cardiovascular dynamics

On the other hand, FFT permits the definition of the exact value of the corresponding frequencies or rhythmic nature. The association of CWT with FFT mathematical methodologies yields a new and very valuable mathematical tool allowing us:

- to establish that the behavior in timefrequency-amplitude of both ventricles and in both arteries is very similar,

- to determine that the frequency of $\mathrm{VE}$ exactly doubled the frequency of the HR in both ventricles,

- to discover that the AM-phenomenon (AM) is present in both ventricles, in the pulmonary artery, and more accentuated in the aorta.

- to visualize the subtle influences of the assisted breathing in both ventricles and that the influence is greater in the pulmonary artery that in the aorta.

- to visualize in separate form the influence of the inspiration and expiration on the pressure of the pulmonary artery. 
The present characterization of the behavior in time-frequency-amplitude of the signals of intra-ventricular pressure and the arteries pulmonary and aorta will permit the study of the modifications that occur in this behavior under different pathological conditions.

\section{ACKNOWLEDGEMENTS}

We thank Sylvia Gutierrez, Cristián Sepúlveda and Jaime Alvarez for their excellent technical assistance. This research was supported by grant 200.013.018-1.0 from the Research Board, University de Concepción. The authors are indebted to the anonymous referee for his very valuable comments regarding the original manuscript which allowed us to improve the comprehension of this very complex subject.

\section{REFERENCES}

ALDROUBI A, UNSER M (1996) Wavelets in Medicine and Biology. Boca Raton, Florida: CRC Press Inc

BEST CH, TAYLOR NB (1985) Physiological Basis of Medical Practice. Baltimore London: Williams \& Wilkins

BURKE MJ, NASOR M (2002) The time relationships of the constituent components of the human electrocardiogram. J Med Eng Technol 26(1), 1-6

CHAN HL, HUANG HH, LIN JL (2001) Time-frequency analysis of heart rate variability during transient segments. Ann Biomed Eng 29 (11): 983-96

CHEN SW (2002) A wavelet-based heart rate variability analysis for the study of nonsustained ventricular tachycardia. IEEE Trans Biomed Eng 49 (7), 736-42
FUNG YC (1984) Biodynamics. Circulation. New York: Springer

GAMERO LG, VILLA J, PALACIOS F (2002) Wavelet transform analysis of heart rate variability during myocardial ischaemia. Med Biol Eng Comput: 40, 72-78

GROSSMANN A, MORLET J (1985) Decomposition of functions into wavelets of constant shape, and related transforms. In: STREIT L (ed.): Mathematics + Physics, Lectures on Recent Results, Vol 1. Singapore: World Scientific

GROSSMANN A, KRONLAND-MARTINET R, MORLET J (1989) Reading and understanding-wavelet transform. In: COMBES JM, GROSSMANN A, TCHAMITCHIAM PH (eds.). Wavelets: Timefrequency Methods and Phase Space. Berlin, Heidelberg: Springer-Verlag. pp: 2-20

GÜNTHER B, JIMÉNEZ RF, PICARTE C (1993) Wavelet analysis of arterial pressure and blood velocity pulsations in the aorta of anesthetized dogs. Biol Res 26: 391-396

GÜNTHER B, JIMÉNEZ R F (1996) Wavelet analysis of aortic pulsations in anesthetized dogs: Effects of spontaneous and positive-pressure respiration. J Biol Syst 4: 45-59

JIMÉNEZ RF (1991) Detections of discontinuities by wavelets transform. In: CARASSO C, CONCA C, CORREA C, PUEL J (eds.) Mathématiques Appliquées aux Sciences de l'Ingénieur. Paris, Cépaduès Editions. pp: 277-304

JIMENEZ RF, GÜNTHER B, SALAZAR A (1997) Continuous wavelet transform of aortic pressure oscillations in anesthetized dogs: Effects of $45^{\circ}$ tilting. Biol Res 30: 53-64

JIMÉNEZ RF, GÜNTHER B, CID L, TORRES P (1999) Pressure oscillations in anesthetized $\operatorname{dogs}$ and its conversion into quasi-periodic orbits. Biol Res 32: 1117

JIMÉNEZ RF, GÜNTHER B, TORRES P (2001) Timefrequency analysis of arterial pressure oscillations in anesthetized dogs: Effects of standardized hemorrhages. Shock 15: 143-150

MOHRMAN DE, HELLER LJ (1981) Cardiovascular Physiology. New York: McGraw-Hill

WIKLUND U, AKAY M, MORRISON S, NIKLASSON U (2002) Wavelet decomposition of cardiovascular signals for baroreceptor function test in pigs. IEEE Trans Biomed Eng 49: 651-661 